\title{
Equivalent Inductance Model for the Design Analysis of Electrodynamic Suspension Coils for the Hyperloop
}

Jungyoul Lim

Korea Railroad Research Institute

Chang-Young Lee

Korea Railroad Research Institute

Ye Jun Oh

Korea Railroad Research Institute

Jeong-Min Jo

Korea Railroad Research Institute

Jin-Ho Lee

Korea Railroad Research Institute

Kwan-Sup Lee

Korea Railroad Research Institute

Suyong Choi ( $\nabla$ suchoi@krri.re.kr)

Korea Railroad Research Institute

\section{Research Article}

Keywords: Equivalent Inductance Model , Design Analysis, Electrodynamic Suspension Coils, Hyperloop

Posted Date: September 24th, 2021

DOl: https://doi.org/10.21203/rs.3.rs-909955/v1

License: (c) (i) This work is licensed under a Creative Commons Attribution 4.0 International License.

Read Full License

Version of Record: A version of this preprint was published at Scientific Reports on December 1st, 2021.

See the published version at https://doi.org/10.1038/s41598-021-02907-7. 


\title{
Equivalent Inductance Model for the Design Analysis of Electrodynamic Suspension Coils for the Hyperloop
}

Jungyoul Lim, Chang-Young Lee, Ye Jun Oh, Jeong-Min Jo, Jin-Ho Lee, Kwan-Sup Lee and Suyong Choi*

New Transportation Innovative Research Center, Korea Railroad Research Institute, Uiwang-si, Gyeonggi-do 16105, Korea

*correspondence: suchoi@krri.re.kr

\begin{abstract}
Hyperloop allows for improved transportation efficiency at higher speeds and a lower power consumption. Various magnetic levitation technologies in existing high-speed maglev trains are being considered to overcome speed limitations for the development of Hyperloop, which are driven inside vacuum tubes at $1,200 \mathrm{~km} / \mathrm{h}$; and superconducting (SC) electrodynamic suspension (EDS) can provide numerous advantages to Hyperloop. such as enabling stable levitation in high-speed driving without control, and increasing the levitation air gap. However, the analysis of the EDS system requires the electromagnetic transient analysis of complex three-dimensional (3D) features, and its computational load generally limits the use of numerical methods, such as the 3D finite element method (FEM) or dynamic circuit theory; This paper presents a novel model that can rapidly and accurately calculate the frequency-dependent equivalent inductance; and it can model the EDS system with the decoupled resistance-inductance (RL) equations of levitation coils. As a design example, the levitation coils of the SC-EDS were designed and analyzed for the Hyperloop, and the results were compared with those of the FEM results to validate the model. In addition, the model was experimentally validated by measuring currents induced by moving pods.
\end{abstract}

\section{Introduction}

Recently, magnetically levitated ultra-high-speed ground transportation, such as Hyperloop, has attracted significant attention worldwide [1-6]. Instead of a traditional wheel-rail system that has a speed limit, noncontact magnetic levitation technologies are being used in high-speed transportation systems. Magnetic levitation technologies for high-speed transport include electromagnetic suspension (EMS), which uses a controlled attractive force between onboard electromagnets and ferromagnetic rails; and electrodynamic suspension (EDS), which uses the induced repulsive force between onboard magnets and conductive rails. The former has been implemented in official lines in Shanghai, China since 2004 [7]; and the latter will be implemented in commercial lines in Japan in 2027 [8]. Furthermore, research was conducted on self-stable magnetic levitation using the flux-pinning effect of high-temperature superconductors (SCs) $[9,10]$.

In Hyperloop that travel at velocities of up to $1,200 \mathrm{~km} / \mathrm{h}$ in a vacuum tube with low air resistance, an active levitation EMS that controls the force transmitted to the steel vacuum tubes or rails may be applied [1113]; or a passive levitation EDS that uses the induced repulsive force acting on onboard magnets moving on conductive rails [14,15]. Hyperloop One [16], which recently succeeded in on-board passenger experiments at velocities of $173 \mathrm{~km} / \mathrm{h}$, switched from EDS to EMS levitation. Each magnetic levitation method has advantages and disadvantages. However, there are several advantages for Hyperloop that shares superconducting electromagnets (SCMs) in the vehicle by EDS levitation and linear synchronous motor 
(LSM) propulsion [17-20]. The strong magnetic field generated by SCMs generates a strong propulsion force to reach subsonic speeds, enables stable levitation in high-speed driving without control, and increases the levitation air gap, which can lower the cost of infrastructure construction. Electrodynamic suspension levitation with SCMs uses an efficient null-flux system with a high lift-to-drag ratio [21-24]. Such null-flux coils are typically arranged on the sidewalls of the guideway to vertically levitate and horizontally guide vehicles with SCMs by induced currents in the coils.

The design and analysis of the EDS system requires an electromagnetic transient analysis of complex threedimensional (3D) features, which can be researched using general numerical methods such as the 3D finite element method (FEM) [25-27]. Although such general numerical methods can achieve accurate results, the transient analysis of a moving vehicle along a linear inductive coil track incurs a high computational load, and is generally limited to the analysis of a particular design or the validation of other analysis models. Therefore, as a more efficient analysis method, the dynamic circuit theory $[28,29]$ is commonly used, which models the electromagnetic elements located in space as time-dependent circuit parameters, and then solves the system equations. The electromagnetic interaction between the SCMs and null-flux coils can be modeled as space- and time-dependent inductances; thus, the EDS system can be directly analyzed by solving the ordinary differential equations (ODE) of resistance-inductance (RL) circuits. However, there are computational loads incurred by the handling of mutual inductance, which is a representation of the magnetic coupling between levitation coils and moving SCMs. For rapid analysis using simplified system models, as achieved in previous studies, only the mutual inductance between electrically connected coils is considered while ignoring the magnetic coupling effect due to adjacent coils [30-32]. In certain cases, only the fundamental waves of the SCM are considered for analysis. However, by ignoring the mutual inductance due to adjacent coils, analysis errors may occur, which can be added or subtracted depending on the coil geometry. For polyphase rotary electrical machines, an inductance matrix, which can cause difficulties in analysis and control, is used by transformation to a magnetically decoupled system based on eigenvalues [33-34]. However, no research has been conducted on the linear arrangement of multiple null-flux coils linearly along an EDS track. A rapid design model [35] was proposed that enables the efficient analysis of the decoupled RL equation of the levitation coils by determining a constant effective inductance that includes all the coupling effects of the adjacent coils. However, accurate analysis data or experimental results are required to extract the effective inductance of the system.

This paper presents an equivalent induction model (EIM) that determines the equivalent inductance of a magnetically decoupled EDS system equation consisting of RL equations for each isolated coil. The EIM utilizes the characteristic of induced current in levitation coils arranged at regular intervals along the guideway, and decoupled RL equations by the EIM can be calculated rapidly and accurately using the Fourier series for the induced electromotive force (EMF). After describing the EIM of the normal and nullflux coils in the EDS system, the levitation coils of the superconducting (SC) EDS were designed and analyzed for the Hyperloop. In addition, to validate the EIM, the analyzed results for various designs were compared with those of the finite element model (FEM); and the induced currents for two types of moving pods, namely, SCMs and permanent magnets (PMs), on a small-scale testbed were compared with the analysis results.

\section{Methodology}

\subsection{Inductance Model for Normal-flux Coils}


The EDS system uses a repulsive force applied to the magnet on the vehicle by the conductive track. As shown in Figure 1, when considering an EDS system in which a magnet is moving at velocity $\boldsymbol{v}$ on levitation coils indexed by $p=\cdots,-1,0,1, \cdots$, currents are induced in the coils to interfere with the movement of the magnet, and the coils magnetize into $\mathrm{N}$ or $\mathrm{S}$ poles by induced currents that produce lift and drag forces acting on the magnet.

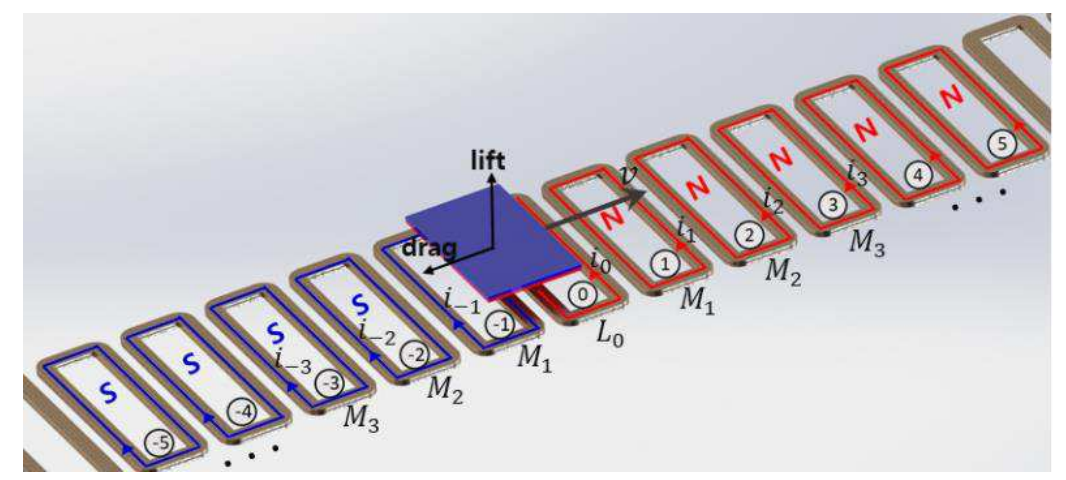

Figure 1. Inductances and induced currents on a discrete EDS coil track, and lift/drag forces acting on a moving magnet.

If an arbitrary coil is selected as the 0 -th coil denoted by $p=0$ in the coil track, it can be considered that other coils are placed at pitch $\tau_{c}$ intervals in both directions from the 0 -th coil. When the $p$-th coil represents a coil located on $p \tau_{c}$ from the 0 -th coil, the pair of the 0 -th and $\pm p$-th coils have the same mutual inductance $M_{p}$. When the magnet is fixed to the origin and the coils move at $-v_{x}$ along the x-direction, the RL equation for the 0 -th coil located at $x=-v_{x} t$ can be expressed as follows:

$$
-v_{x} L_{0} \frac{d}{d x} i_{0}(x)-v_{x} \sum_{p=1}^{\infty}\left(M_{p} \frac{d}{d x}\left(i_{p}+i_{-p}\right)\right)+R i_{0}=\varepsilon_{0}
$$

where $i_{p}$ and $\varepsilon_{p}$ denote the induced current and electromotive force (EMF) at the $p$-th coil. For the identical resistance $R$ and self-inductance $L_{0}$ for each coil, the induced EMF and current of the $p$-th coil, which are caused by the relative motion between the magnet and coil, have the following characteristics of position difference with respect to the 0 -th coil:

$$
\begin{aligned}
& \varepsilon_{p}(x)=\varepsilon_{0}\left(x+p \tau_{c}\right) \\
& i_{p}(x)=i_{0}\left(x+p \tau_{c}\right) .
\end{aligned}
$$

When an even number of onboard magnets are paired with the north $(\mathrm{N})$ and south $(\mathrm{S})$ poles, in which case the induced EMF of the 0 -th coil $\varepsilon_{0}$ is an even function. For coil position $x$ in the $2 \tau_{0}$ interval, the Fourier frequency $\omega_{n}$ is defined as follows:

$$
\omega_{n}=-\pi n / \tau_{0}
$$

With the Fourier coefficient $A_{n}, \varepsilon_{0}$ can be represented by the Fourier cosine series in Equation (4). In the case of an odd number of onboard magnets, the odd function $\varepsilon_{0}$ can be expressed as a Fourier sine series:

$$
\varepsilon_{0}(x)=\sum_{n=1}^{\infty} \varepsilon_{0 n} \text { where } \varepsilon_{0 n}=v_{x} A_{n} \cos \left(\omega_{n} x\right) .
$$

In addition, the induced current at the $p$-th coil can be expressed in the general form of the Fourier series for the coefficients $B_{n}$ and $C_{n}$ : 


$$
i_{p}(x)=\sum_{n=1}^{\infty} i_{p n} \text { where } i_{p n}=B_{n} \cos \left(\omega_{n}\left(x+p \tau_{c}\right)\right)+C_{n} \sin \left(\omega_{n}\left(x+p \tau_{c}\right)\right) .
$$

Using Equation (5), the sum of induced currents of the $\pm p$-th coil pair can be explicitly expressed in relation to the induced current on the 0 -th coil $i_{0 n}$ :

$$
i_{p n}+i_{-p n}=2 \cos \left(\omega_{n} p \tau_{c}\right) i_{0 n}
$$

By applying Equation (6) to Equation (1), the LR equation of the $n$-th harmonic for the 0 -th coil can be obtained as follows:

$$
-v_{x}\left(L_{0}+2 \sum_{p=1}^{\infty} M_{p} \cos \left(\omega_{n} p \tau_{c}\right)\right) \frac{d}{d x} i_{0 n}+R i_{0 n}=\varepsilon_{0 n}
$$

where the 0 -th coil is magnetically decoupled with other coils. Therefore, considering that mutual inductance decreases rapidly with distance, the magnetic effects of adjacent coils at a specific $n$-th harmonic can be replaced by the equivalent inductance $L_{e}$ in Equation (8), or explicitly represented by the ratio of interval $2 \tau_{0}$ and coil pitch $\tau_{c}$ using Equation (3).

$$
L_{e}(n)=L_{0}+\sum_{p=1}^{P} 2 M_{p} \cos \left(\omega_{n} p \tau_{c}\right)=L_{0}+\sum_{p=1}^{P} 2 M_{p} \cos \left(p n \pi \frac{\tau_{c}}{\tau_{0}}\right)
$$

where $P_{e}$ is the number of pairs of adjacent coils to be included in $L_{e}$, and $L_{e}=L_{0}$ for $P_{e}=0$. Moreover, $L_{0}$ and $M_{p}$ can be readily calculated by static analysis using various numerical methods; and with the decoupled LR equation (7), the induced current of the $p$-th coil $i_{p n}$ expressed in closed-form solutions [35] can be calculated directly as follows:

$$
i_{p n}=-\frac{A_{n} v_{x}}{\sqrt{R^{2}+\left(v_{x} \omega_{n} L_{e}\right)^{2}}} \sin \left(\omega_{n} x+\varphi_{p}+\varphi_{n}\right)
$$

where $\varphi_{p}=\omega_{n} p \tau_{c}$ and $\varphi_{\mathrm{n}}=\tan ^{-1} \frac{\mathbf{v}_{\mathbf{x}} \omega_{\mathrm{n}} \mathrm{L}_{\mathrm{n}}}{\mathrm{R}}$ represent the phase shifts due to the $p$-th coil position and coil inductance, respectively.

\subsection{Inductance Model for Null-flux Coils}

Using the EIM for normal flux coils, the equivalent inductance on a null-flux EDS track can be readily determined. Figure 2 presents the null-flux coils attached to the sidewall and a moving SCM pod, which has two SCMs consisting of two poles with a current $i_{S C M}$. A set of null-flux coils composed of four coils placed on both sidewalls are electrically connected, such that all induced EMFs are canceled at the center, thus resulting in induced EMFs and restoring forces proportional to the vertical and horizontal displacements from the center. As in the case of normal flux coils, the arbitrarily selected 0 -th coil and the $p$-th coil located relatively on $p \tau_{c}$ from the 0 -th coil can be considered. The four coils of the $p$-th null-flux coil are numbered by subscripts $k=1-4$ from the top left to the bottom right, and $\varepsilon_{p_{k}}$ and $i_{p_{k}}$ denote the induced EMF and current of the $p_{k}$-th coil, respectively. 


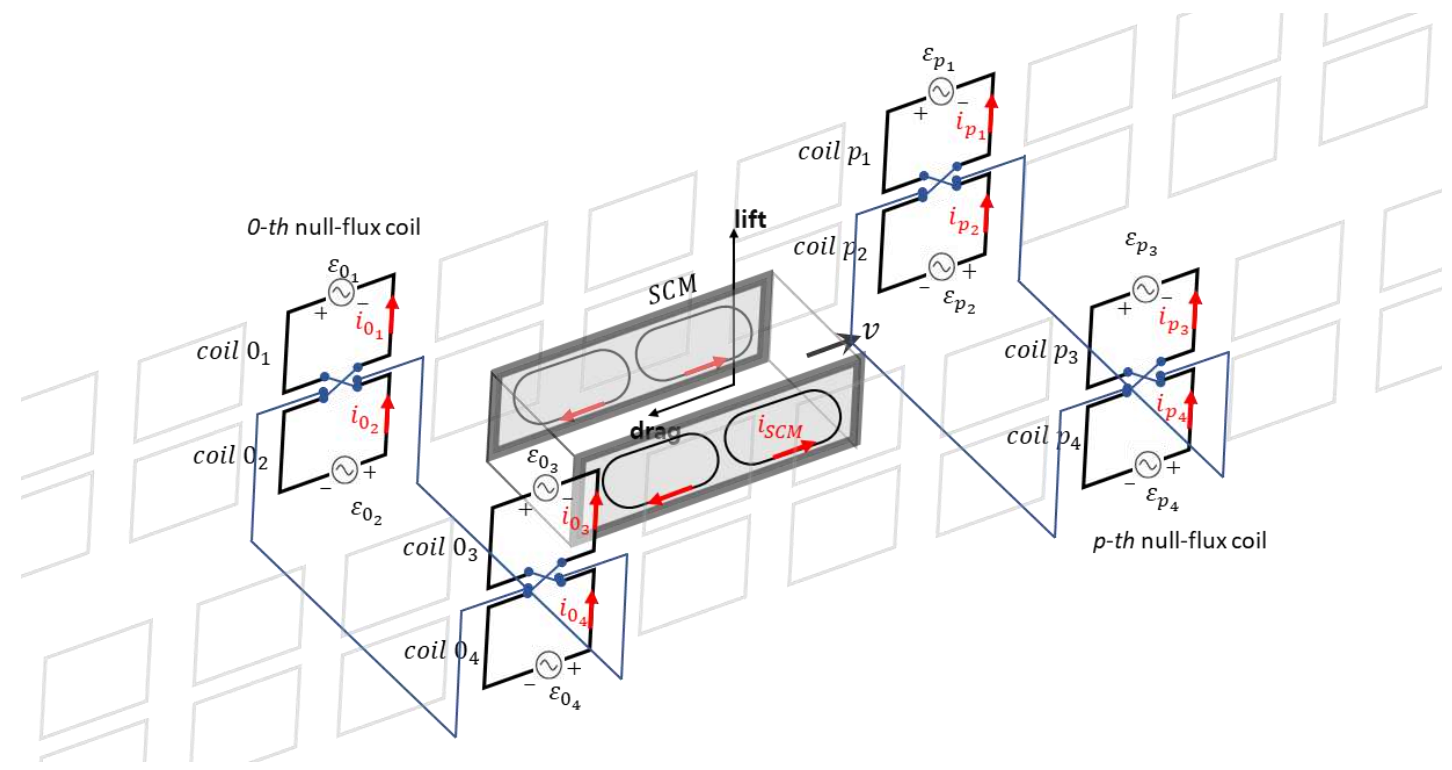

Figure 2. Schematic diagram of the SCM pod moving on the null-flux EDS track, and configuration of the null-flux coil consisting of four electrically connected coils.

The coils on the same sidewall are situated close by and magnetically influence each other, while the magnetic interaction of the coils located on both sidewalls can be ignored. Considering coils 0 and $p$ on the same sidewall, the mutual inductance between the coils at the same and different vertical positions can be respectively represented by $M_{p a}$ and $M_{p b}$. When the self-inductance of a coil is $L_{s}$ and the mutual inductance between electrically connected vertical coils is $M_{0}$, the RL equation for the 0 -th null-flux coil has a similar form to Equation (1) for mutual and self-inductance matrices $\mathbf{M}$ and $\mathbf{L}$ as follows:

$$
-v_{x} \mathbf{L} \frac{d}{d x} \boldsymbol{i}_{0}-v_{x} \sum_{p=1}^{\infty} \mathbf{M} \frac{d}{d x}\left(\boldsymbol{i}_{p}+\boldsymbol{i}_{-p}\right)+R \boldsymbol{i}_{0}=\boldsymbol{\varepsilon}_{0}
$$

where the induced current vector $\boldsymbol{i}_{p}=\left[\begin{array}{llll}i_{p_{1}} & i_{p_{2}} & i_{p_{3}} & i_{p_{4}}\end{array}\right]^{T}$; the induced EMF vector $\boldsymbol{\varepsilon}_{0}=$ $\left[\begin{array}{llll}\varepsilon_{0_{1}} & \varepsilon_{0_{2}} & \varepsilon_{0_{3}} & \varepsilon_{0_{4}}\end{array}\right]^{T} \quad ; \quad \mathbf{M}=\left[\begin{array}{cc}\boldsymbol{M}_{\boldsymbol{p}} & \boldsymbol{O}_{2} \\ \boldsymbol{O}_{2} & \boldsymbol{M}_{\boldsymbol{p}}\end{array}\right] ;$ and $\boldsymbol{M}_{\boldsymbol{p}}=\left[\begin{array}{ll}M_{p a} & M_{p b} \\ M_{p b} & M_{p a}\end{array}\right] \quad$. Moreover, $\quad \mathbf{L}=$ $\left[\begin{array}{ll}\boldsymbol{L}_{\mathbf{0}} & \boldsymbol{O}_{2} \\ \boldsymbol{O}_{2} & \boldsymbol{L}_{\mathbf{0}}\end{array}\right]$, where $\boldsymbol{L}_{\mathbf{0}}=\left[\begin{array}{cc}L_{S} & 0 \\ 0 & M_{0}\end{array}\right]$ and $\boldsymbol{O}_{2}$ is a $2 \times 2$ zero matrix. For each sidewall on the $p$-th null-flux coil, $\boldsymbol{i}_{p}$ can be expressed separately by the current $\boldsymbol{i}_{p L}$, which is equal in size and opposite in direction; and $\boldsymbol{i}_{p G}$, which is equal in size and direction.

$$
\boldsymbol{i}_{p}=\boldsymbol{i}_{p L}+\boldsymbol{i}_{p G}
$$

where $\boldsymbol{i}_{p_{L}}=\left[\begin{array}{lllll}i_{p_{a} L} & -i_{p_{a} L} & i_{p_{b} L} & -i_{p_{b} L}\end{array}\right]^{T} ; \quad \boldsymbol{i}_{p G}=\left[\begin{array}{llll}i_{p_{a} G} & i_{p_{a} G} & i_{p_{b} G} & i_{p_{b} G}\end{array}\right]^{T} ; i_{p_{a} L}=\left(i_{p_{1}}-i_{p_{2}}\right) / 2 ;$ $i_{p_{b} L}=\left(i_{p_{3}}-i_{p_{3}}\right) / 2 ; i_{p_{a} G}=\left(i_{p_{1}}+i_{p_{2}}\right) / 2$; and $i_{p_{b} G}=\left(i_{p_{3}}+i_{p_{3}}\right) / 2$. In addition, applying Kirchhoff's current law to any node yields the following:

$$
i_{p_{a} G}=-i_{p_{b} G}
$$

The equation for $\boldsymbol{i}_{p L}$ is obtained by applying Equation (11) to Equation (10), and then removing $\boldsymbol{i}_{p G}$ by subtracting rows, which yields the following: 


$$
-v_{x}\left(L_{s}-M_{0}\right) \frac{d}{d x} \boldsymbol{i}_{0 L}-v_{x} \sum_{p=1}^{\infty}\left(M_{p a}-M_{p b}\right) \frac{d}{d x}\left(\boldsymbol{i}_{p L}+\boldsymbol{i}_{-p L}\right)+R \boldsymbol{i}_{0 L}=\boldsymbol{\varepsilon}_{0 L}
$$

where $\boldsymbol{\varepsilon}_{0 L}=\mathbf{A}_{L} \boldsymbol{\varepsilon}_{0}$ denotes induced EMF for $\boldsymbol{i}_{p L} ;$ and $\mathbf{A}_{L}=\frac{1}{2}\left[\begin{array}{rrrr}1 & -1 & 0 & 0 \\ -1 & 1 & 0 & 0 \\ 0 & 0 & 1 & -1 \\ 0 & 0 & -1 & 1\end{array}\right]$.

From $\boldsymbol{\varepsilon}_{0 L}, \boldsymbol{i}_{p L}$ induced by the EMF difference between the cross-connected vertical coils generates a levitation force to the SCM pod. Comparing Equations (13) and (1), $L_{0}=L_{s}-M_{0}$ and $M_{p}=M_{p a}-M_{p b}$, and the equivalent inductance $L_{e L}$ is expressed as follows:

$$
L_{e L}(n)=\left(L_{s}-M_{0}\right)+\sum_{p=1}^{P} 2\left(M_{p a}-M_{p b}\right) \cos \left(p n \pi \frac{\tau_{c}}{\tau_{0}}\right) .
$$

In addition, $\boldsymbol{i}_{p G}$ is obtained by applying Equations (11) and (12) to Equation (10), and then removing $\boldsymbol{i}_{p L}$ by adding rows, which yields the following:

$$
-v_{x}\left(L_{s}+M_{0}\right) \frac{d}{d x} \boldsymbol{i}_{0 G}-v_{x} \sum_{p=1}^{\infty}\left(M_{p a}+M_{p b}\right) \frac{d}{d x}\left(\boldsymbol{i}_{p G}+\boldsymbol{i}_{-p G}\right)+R \boldsymbol{i}_{0 G}=\boldsymbol{\varepsilon}_{0 G}
$$

where $\boldsymbol{\varepsilon}_{0 G}=\mathbf{A}_{G} \boldsymbol{\varepsilon}_{0}$ denotes the induced EMF for $\boldsymbol{i}_{p G}$, and $\mathbf{A}_{G}=\frac{\mathbf{1}}{\mathbf{4}}\left[\begin{array}{rrrr}1 & 1 & -1 & -1 \\ 1 & 1 & -1 & -1 \\ -1 & -1 & 1 & 1 \\ -1 & -1 & 1 & 1\end{array}\right]$.

Similarly, from $\varepsilon_{0 G}, \boldsymbol{i}_{p G}$ induced by the EMF difference between null-flux connected horizontal coils generates a guidance force to the SCM pod. Comparing Equations (15) and (1), $L_{0}=L_{s}+M_{0}, M_{p}=$ $M_{p a}+M_{p b}$, and the equivalent inductance $L_{e G}$ is expressed as follows:

$$
L_{e G}(n)=\left(L_{s}+M_{0}\right)+\sum_{p=1}^{P} 2\left(M_{p a}+M_{p b}\right) \cos \left(p n \pi \frac{\tau_{c}}{\tau_{0}}\right) .
$$

Therefore, $\boldsymbol{i}_{p L}$ and $\boldsymbol{i}_{p G}$ are readily determined by applying $\boldsymbol{\varepsilon}_{0 L}, \boldsymbol{\varepsilon}_{0 G}, L_{e L}$, and $L_{e G}$ to Equation (9), and the induced current $\boldsymbol{i}_{p}$ can be obtained by summing the two currents, as expressed by Equation (11).

2.3 Induced EMF and Force Equation for EDS Coils

When magnets move at $\boldsymbol{v}=\left[\begin{array}{lll}v_{x} & v_{y} & v_{z}\end{array}\right]^{T}$, coils can be considered to move at $-\boldsymbol{v}$ instead of magnets. In this case, the induced EMF $\varepsilon$ and force $\boldsymbol{f}_{(\mathrm{lev})}$ on a coil can be determined by the induced current $I$ and EMF vector $\boldsymbol{b}[29,35]$ :

$$
\begin{gathered}
\varepsilon=\boldsymbol{b}^{T} \boldsymbol{v} \\
\boldsymbol{f}_{(l e v)}=\boldsymbol{b} I
\end{gathered}
$$

where $\boldsymbol{b}=\left[\begin{array}{lll}b_{(x)} & b_{(y)} & b_{(z)}\end{array}\right]^{T}$ is defined by Faraday's law for moving conductors. For the unit directional vector $\boldsymbol{e}_{(d)}(d=x, y, z)$, a line integral following Wire loop $\mathrm{C}$ on the coil yields $b_{(d)}$ as follows:

$$
b_{(d)}=-\oint_{C} \boldsymbol{e}_{(d)} \times \boldsymbol{B}(\boldsymbol{r}) \cdot d \boldsymbol{l}
$$

where $\boldsymbol{B}(\boldsymbol{r})$ is the flux density of the magnet at the wire position $\mathbf{r}$; and $\mathrm{d} \boldsymbol{l}$ is an infinitesimal vector element of Wire loop C. 
Based on the force acting on the moving coil, as expressed by Equation (18), the total force acting on the moving magnet can be calculated by the sum of the forces acting on the $k$-th coil of the $p$-th null-flux coil when the EDS track consists of $2 P_{0}+1$ null-flux coils.

$$
\boldsymbol{f}=-\sum_{p=-P_{0}}^{P_{0}} \sum_{k=1}^{4} \boldsymbol{f}_{p_{k}(l e v)}
$$

For a more rapid calculation of the $\boldsymbol{b}$, virtual $n_{\text {turn }}=n_{w} \times n_{t}$ turns of Closed loop $C_{i}$ are considered instead of actual wire loops with $N_{t u r n}=N_{w} \times N_{t}$ turns. Thereafter, $b_{(d)}$ can be approximated by the sum of $b_{i(d)}$ calculated for each $C_{i}$ as follows:

$$
b_{(d)} \approx\left(N_{t u r n} / n_{t u r n}\right) \sum_{i=1}^{n_{t u r n}} b_{i(d)}
$$

In addition, when $C_{i}$ is divided into $n_{i j}$ small line segments, the induced EMF vector $b_{i}$ of $C_{i}$ can be approximated by the Riemann sum as follows:

$$
b_{i(d)} \approx \sum_{j=1}^{n_{i j}} \boldsymbol{e}_{(d)} \times \boldsymbol{B}\left(\boldsymbol{r}_{i j}\right) \cdot \boldsymbol{l}_{i j}
$$

where $\boldsymbol{r}_{i j}$ and $\boldsymbol{l}_{i j}$ denote the center position and length of the $j$-th segment on $C_{i}$, respectively.

\section{Results}

To demonstrate the effectiveness of the EIM, null-flux levitation coils for small-scale hyperloop testbeds were designed. After describing the design parameters for the SCM and null-flux coils used in the design, the results of the performance analysis for various designs are presented. Three designs were selected based on the performance criteria; and detailed performance metrics such as the magnetic force and stiffness were compared under various operating conditions, such as the pod mass and velocity. Furthermore, the results obtained using the EIM were compared with the results obtained by an FEM, to validate the accuracy of the model. The magnetic field of the magnet, inductance of the coil, and analysis results for validation were computed using Simcenter MagNet, which is a commercial FEM software.

\subsection{Design Parameters of EDS Coils for the Hyperloop}

Figure 3 illustrates the concept of Hyperloop in which a hyperloop vehicle travels at subsonic speeds along an electromagnetic guideway in a vacuum tube. A pair of SCMs is mounted on both sides of the pod supporting the Hyperloop vehicle, and the SCM pod is driven in the x-direction by the LSM installed on the guideway. In addition, the pod is levitated and guided by null-flux EDS on the guideway, which generates restoring lift and guidance forces in proportion to vertical $\mathrm{z}$ - and horizontal y-directional displacements. For a small-scale testbed of the Hyperloop, we designed null-flux coils to illustrate the effectiveness of the EIM.

Figure 4 presents the design layout of the small-scale SCM module and null-flux levitation coil for industrial applications. For the testbed, each module of the SCM, consisting of two poles separated by pole pitch $\tau_{S C M}$, was set to the center size of $L_{(S C M) x} \times L_{(S C M) z}$ and magnetomotive force (MMF). Each nullflux coil was separated by pitch $\tau_{c}$, the center size of the coil was $L_{x c} \times L_{z c}$, and the coil gap along the moving direction was $d_{x}$. In addition, the multi-turn null-flux coil had a number of turns $N_{t u r n}$, which consisted of $N_{w}$ turns in the xz plane and $N_{t}$ turns along the y-axis with wire thicknesses $c_{w}$ and $c_{t}$, respectively. The detailed design parameters and variables are summarized in Table 1. Given that the physical air gap was $g_{\text {air }}$ and the thickness of the levitation coil was $L_{t}$, the performance of the levitation 
coil design was evaluated at subsonic driving velocity $v_{h}$ and/or the take-off velocity $v_{l}$. Based on these design layouts and parameters, the performances of various $N_{t u r n}$ and $L_{z c}$ values were analyzed for three horizontal pitches with high performances, and the design with the preferred performance for each pitch was selected and analyzed.

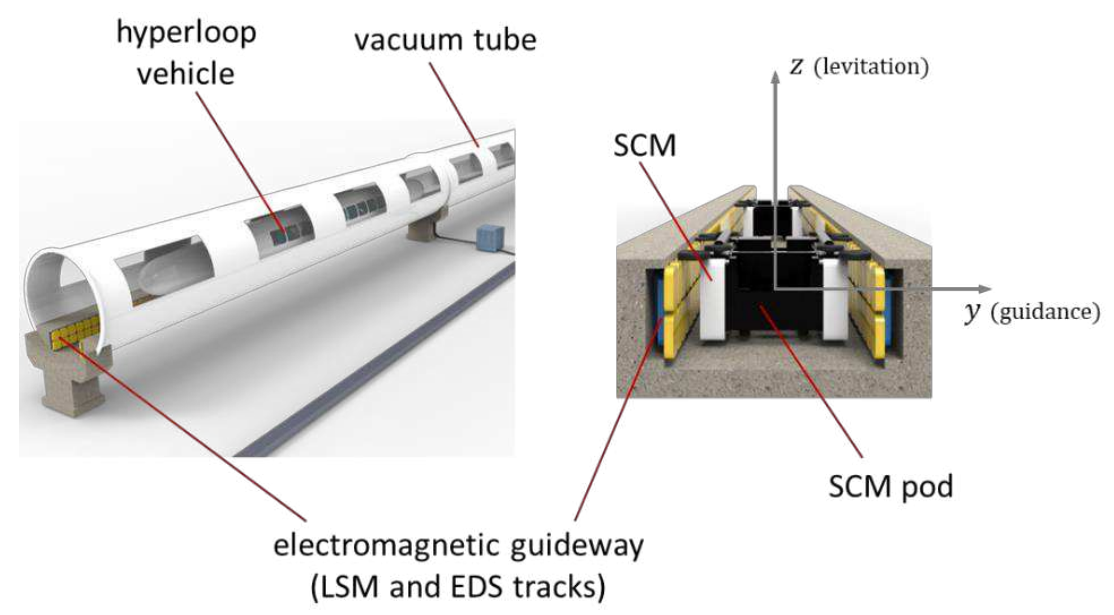

Figure 3. Concept of the hyperloop vehicle with SCM pods in a vacuum tube with an electromagnetic guideway consisting of LSM and EDS tracks.

Table 1. Parameters of SCM and null-flux levitation coils for design examples and model validation.

\begin{tabular}{|c|c|c|c|}
\hline & Parameter & Value & Unit \\
\hline \multirow{3}{*}{$\mathrm{SCM}$} & Pole pitch, $\tau_{S C M}$ & 0.81 & $\mathrm{~m}$ \\
\hline & Coil size, $L_{(S C M) x} \times L_{(S C M) z}$ & $0.6 \times 0.3$ & $\mathrm{~m}^{2}$ \\
\hline & Magnetomotive force & 300 & kAt \\
\hline \multirow{14}{*}{$\begin{array}{l}\text { levitation } \\
\text { coil }\end{array}$} & Number of turns, $N_{\text {turn }}=N_{w} \times N_{t}$ & $N_{t}=2, N_{w}=2, \ldots, 15$ & - \\
\hline & Horizontal pitch, $\tau_{c}$ & $(1 / 3,1 / 2,2 / 3) \times \tau_{S C M}$ & $\mathrm{~m}$ \\
\hline & Vertical coil height, $L_{z c}$ & $0.2,0.21, \ldots, 0.39,0.4$ & $\mathrm{~m}$ \\
\hline & Wire cross sectional area, $A_{w}=c_{w} \times c_{t}$ & $0.01 \times 0.01$ & $\mathrm{~m}^{2}$ \\
\hline & Horizontal gap between coils, $d_{x}$ & 0.03 & $\mathrm{~m}$ \\
\hline & Horizontal coil width, $L_{x c}$ & $\tau_{c}-d_{x}-c_{w} N_{w}$ & $\mathrm{~m}$ \\
\hline & Vertical pitch, $\tau_{z}$ & $L_{z c}+c_{w} N_{w}$ & $\mathrm{~m}$ \\
\hline & Aluminum wire resistivity, $\rho_{\text {alu }}$ & $2.8265 \times 10^{-8}$ & $\Omega \cdot \mathrm{m}$ \\
\hline & Air gap, $g_{\text {air }}$ & 0.05 & $\mathrm{~m}$ \\
\hline & Thickness of the levitation coil, $L_{t}$ & 0.03 & $\mathrm{~m}$ \\
\hline & Subsonic driving velocity, $v_{h}$ & $277.78(1,000)$ & $\mathrm{m} / \mathrm{s}(\mathrm{km} / \mathrm{h})$ \\
\hline & Take-off velocity, $v_{l}$ & $41.67(150)$ & $\mathrm{m} / \mathrm{s}(\mathrm{km} / \mathrm{h})$ \\
\hline & Maximum vertical displacement, $\Delta z_{\max }$ & 0.10 & $\mathrm{~m}$ \\
\hline & Maximum horizontal displacement, $\Delta y_{\max }$ & 0.05 & $\mathrm{~m}$ \\
\hline
\end{tabular}




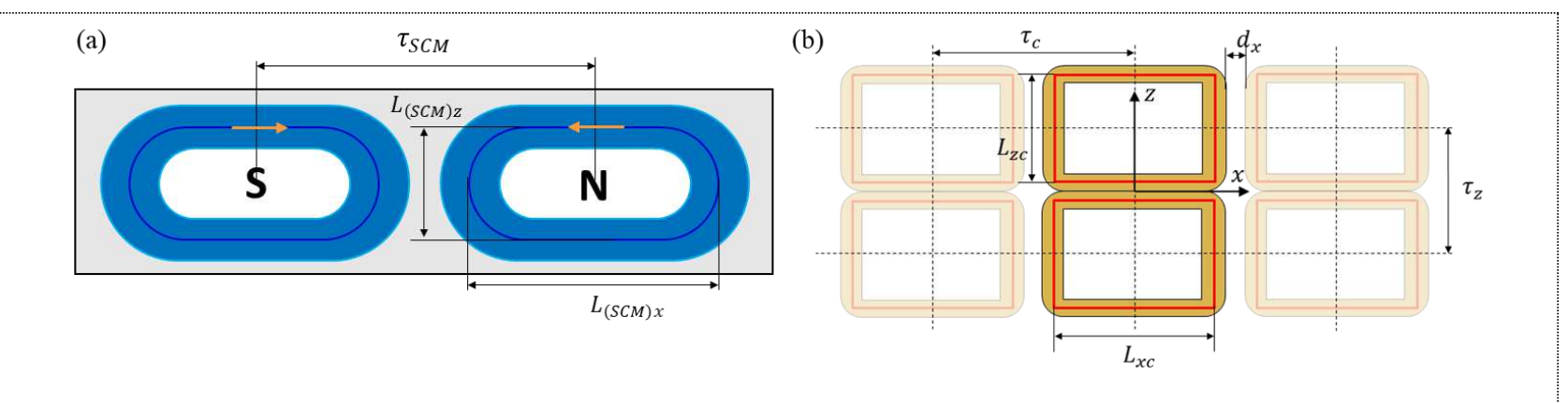

Figure 4. Design layouts of the (a) SCM module and (b) null-flux coil for the design examples and model validation.

\subsection{Design Results for Null-flux EDS Coils}

The analysis results for the lift force $F_{z}$ and horizontal stiffness $k_{y}$ of three types of levitation coil pitches with respect to changes in $N_{t u r n}$ and $L_{z c}$ at $v_{h}$ are shown in Figure 5. To represent each pair of horizontal and vertical displacements as $(\Delta \mathrm{y}, \Delta \mathrm{z}), F_{z}$ and $k_{y}$ were evaluated at the maximum vertical displacement $P_{z m}=\left(0,-\Delta z_{\max }\right)$ and the intermediate vertical displacement $P_{z h}=\left(0,-\Delta z_{\max } / 2\right)$, respectively. A simple method for selecting a design is to maximize the lift force indicated by the black dots on each graph; however, such selections can lead to an excessively low horizontal stiffness [36]. If a gentle characteristic of the $F_{z}$ surface near the peak point is utilized, it can compensate for the low horizontal stiffness by reducing $F_{z}$ to a certain level. Therefore, if the maximum $F_{z}$ of each pole pitch is $F_{z m}$ and the acceptable $F_{z}$ reduction ratio is $\alpha_{z m}$, a design that maximizes the horizontal stiffness with greater $F_{z}$ values than (1$\left.\alpha_{z m}\right) F_{z m}$, as indicated by the green dots, can be selected from each pitch. Considering that $F_{z m}$ is significantly different for each pitch ratio, $\alpha_{z m}$ was applied differently by $0.02,0.06$, and 0.10 . The newly selected designs in Figure 5 are indicated by a red dot, where $L_{z c}$ or the vertical pitch $\tau_{z}$ was reduced; and $N_{\text {turn }}$ was reduced for several designs. Thus, when Designs A0, B0, and C0, as indicated by black dots, were the original designs that maximized $F_{z}$ at each pole pitch; Designs A1, B1, and C1, as indicated by red dots, were the improved designs that enhanced the horizontal stiffness from each original design, respectively. The shape parameters and performance of the design are summarized in Table 2 . In all cases, $\tau_{z}$ was selected with the same value. For each pole pitch, Designs A1, B1, and C1 demonstrated considerably improved horizontal stiffnesses to $22.7 \%, 41.6 \%$, and $56.4 \%$ at $P_{z h}$ instead of only $1.7 \%$, $5.6 \%$, and $9.0 \% F_{z}$ reductions at $P_{z m}$, respectively. In addition, the levitation and guidance performances of $\mathrm{B} 1$ and $\mathrm{C} 1$ were similar.

Table 2. Comparison of parameters and performances between designs that maximize the levitation force and designs with improved horizontal stiffness.

\begin{tabular}{ccccccc}
\hline Design & $\boldsymbol{\tau}_{\boldsymbol{c}} / \boldsymbol{\tau}_{\boldsymbol{S C M}}$ & $\boldsymbol{N}_{\text {turn }}$ & $\boldsymbol{L}_{\boldsymbol{z c}}(\mathbf{m})$ & $\boldsymbol{\tau}_{\boldsymbol{z}}(\mathbf{m})$ & $\boldsymbol{F}_{\boldsymbol{z}}(\mathbf{k N})$ at $\boldsymbol{P}_{\boldsymbol{z m}}$ & $\boldsymbol{k}_{\boldsymbol{y}}(\mathbf{k N} / \mathbf{m})$ at $\boldsymbol{P}_{\boldsymbol{z h}}$ \\
\hline $\mathrm{A} 0$ & \multirow{2}{*}{$1 / 3$} & 12 & 0.30 & 0.36 & 18.46 & 171.76 \\
$\mathrm{~A} 1$ & & 10 & 0.28 & 0.33 & 18.15 & 210.81 \\
B0 & \multirow{2}{*}{$1 / 2$} & 18 & 0.29 & 0.38 & 22.44 & 175.11 \\
B1 & & 18 & 0.24 & 0.33 & 21.18 & 247.91 \\
C0 & \multirow{2}{*}{$2 / 3$} & 24 & 0.27 & 0.39 & 23.56 & 163.21 \\
$\mathrm{C} 1$ & & 22 & 0.22 & 0.33 & 21.44 & 255.32 \\
\hline
\end{tabular}



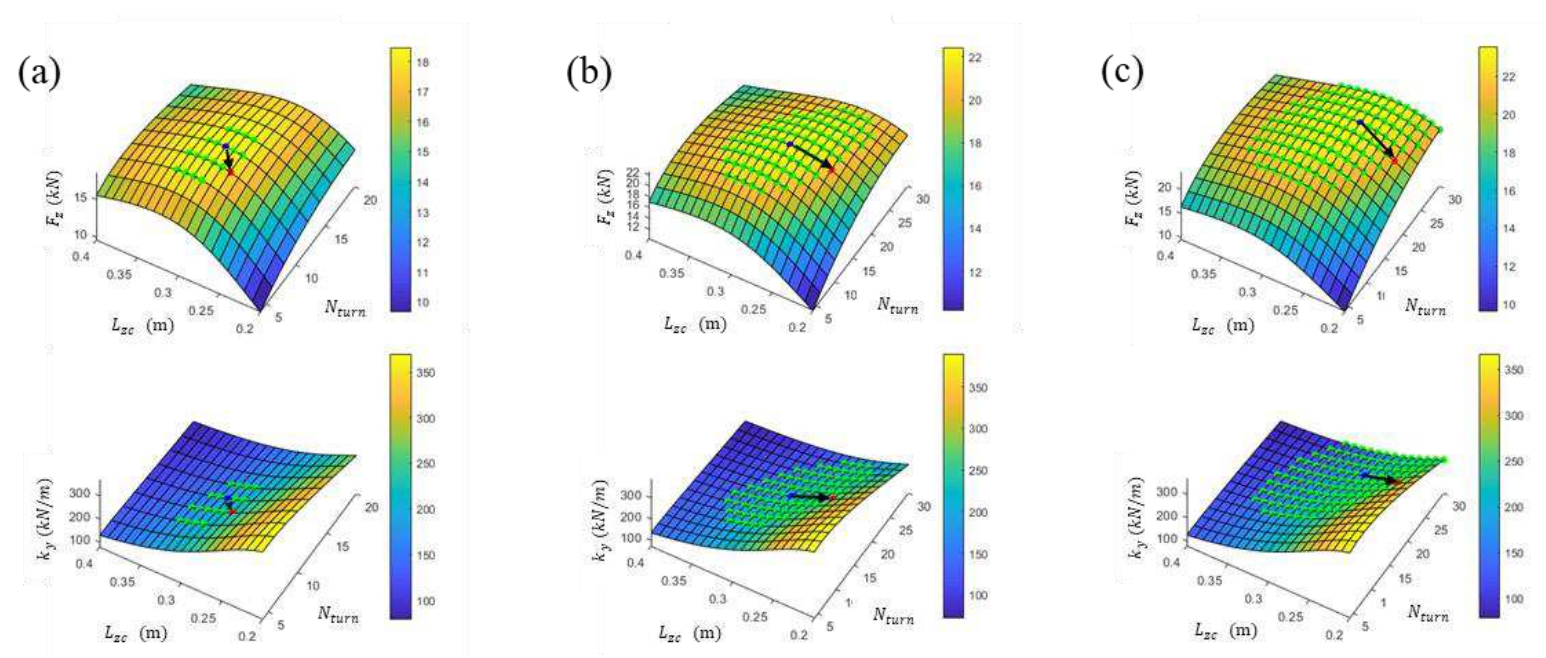

Figure 5. Design of null-flux coils with analyzed lift force at $P_{z m}$ and guidance stiffness at $P_{z h}$ with respect to changes in $N_{t u r n}$ and $L_{z c}$ at the selected pole pitches: (a) $\tau_{c}=(1 / 3) \tau_{S C M}$, (b) $\tau_{c}=(1 / 2) \tau_{S C M}$, and (c) $\tau_{c}=(2 / 3) \tau_{S C M}$.

The performances and characteristics of the improved designs of A1, B1, and C1 were analyzed and compared in detail. First, when driving at velocity $v_{h}$, as shown in Figure 6, the lift and drag forces and horizontal and vertical stiffness according to the vertical displacement at the driving horizontal center $(\Delta y=$ 0 ) were compared. Designs B1 and C1 demonstrated highly similar performances; however, the forces and stiffnesses of Design A1 were relatively lower at the same value of $\Delta \mathrm{z}$. As the vertical displacement increased, the lift force gradually increased. However, the vertical stiffness defined by the slope of the lift force decreased gradually, thus resulting in a maximum of $\Delta \mathrm{z}=-85 \sim 90 \mathrm{~mm}$. There was a gradual increase in the drag force and horizontal stiffness initially as $\Delta z$ decreased, and then a gradual increase to a considerably high value. To analyze the driving characteristics of a design in terms of the lift-to-drag (LD) ratio, for a small vertical displacement, the LD ratio should be high, thus allowing for a higher driving efficiency based on magnetic levitation. For large vertical displacements, the LD ratio should be low, which results in a lower driving efficiency. To compare the performance of a more detailed design, the performance under conditions in which lightweight pods of the same weight are driven by magnetic levitation were first compared, followed by pods of different weights that can be magnetically levitated at the same vertical displacement.

First, when the lightweight pod mass was set as 1.35 ton, each design generated a required $13.24 \mathrm{kN}$ of levitation force at different vertical displacements. The drag and stiffness corresponding to the vertical displacement are represented in Figure 6 as blue, red, and black circles; and are summarized in Table 3. Designs C1 and B1 generated higher lift forces than Design A1, thus confirming that pods can be levitated from a smaller vertical displacement. Therefore, the LD ratio was higher, which implies that driving based on magnetic levitation is more efficient than Design A1. Although Design A1 with similar vertical and horizontal stiffnesses can be relatively stable, Designs C1 and B1 have relatively low horizontal stiffnesses, which may be relatively weak to horizontal guideway irregularity.

Thereafter, the characteristics of the three designs at the same vertical displacement were compared. For $\Delta \mathrm{z}=-50 \mathrm{~mm}$, the lift and drag forces and horizontal and vertical stability according to changes in the driving velocity were compared, as shown in Figure 7 and Table 4, where the lift force and vertical/horizontal stiffness gradually increased with an increase in velocity, and then converged to a constant value. Moreover, the drag force resulted in an abrupt increase from a low to maximum rate at $40-60 \mathrm{~km} / \mathrm{h}$, and then decreased 
rapidly with an increase in velocity. The performances of Designs B1 and C1 in all performance aspects of levitation/guidance were similar, with the $F_{z}, k_{y}$ and LD ratio of Design $\mathrm{C} 1$ slightly superior. However, under the given SCMs and driving conditions, the performance of Design A1 was relatively inferior in all respects. The acceptable lifting weights of the three designs were 1.41 tons, 1.76 tons, and 1.83 tons, respectively; and the sizes or MMFs of the SCMs should be increased to allow for the on-boarding of passengers. To compare the increase rate of the lift force at low velocities, the ratios of lift forces at $v_{l}$ and $v_{h}$ denoted by $F_{z r}$ were compared in Table 4; in which the rates of $F_{z r}$ of Designs B1 and C1 were high, and that of A1 was relatively low, which can be improved by reducing the resistance, e.g., by using thicker wires or lower resistivity materials.
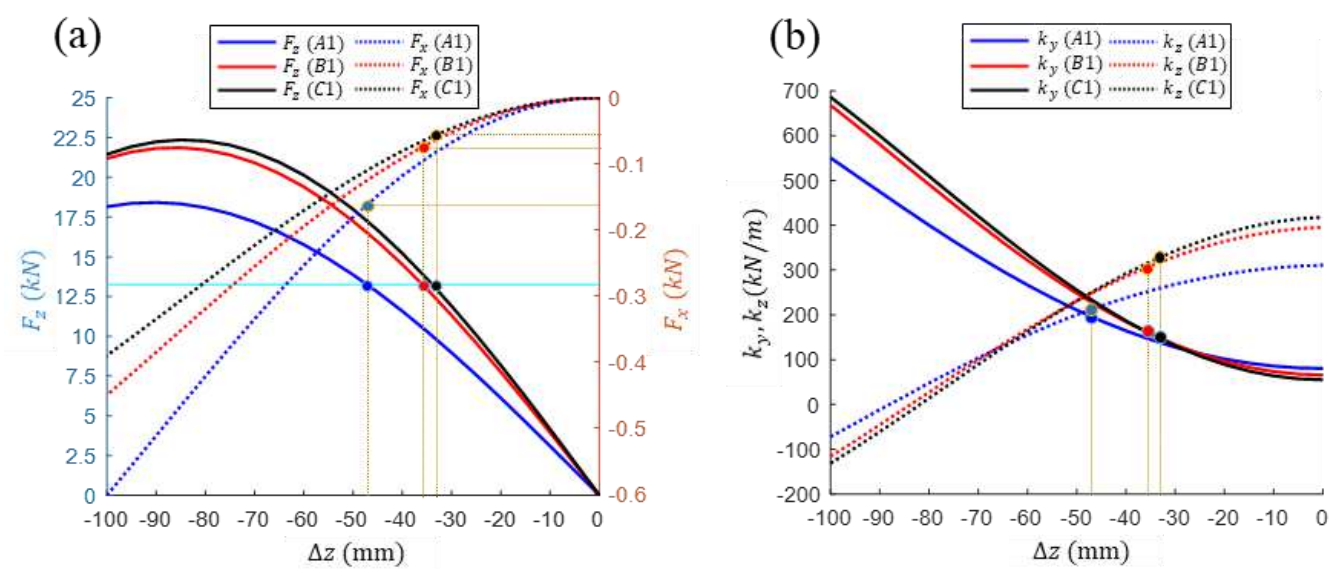

Figure 6. Comparison of characteristics of designs with respect to vertical displacement; (a) lift and drag forces; and (b) horizontal and vertical stiffnesses.

Table 3. Comparison of magnetic levitation/guidance characteristics of three designs for a pod with mass of 1.35 tons moving at velocity $v_{h}$.

\begin{tabular}{ccccccc}
\hline Design & $\Delta \boldsymbol{z}(\mathbf{m m})$ & $\boldsymbol{F}_{\boldsymbol{z}}(\mathbf{k N})$ & $\boldsymbol{F}_{\boldsymbol{x}}(\mathbf{k N})$ & $\boldsymbol{k}_{\boldsymbol{y}}(\mathbf{k N} / \mathbf{m})$ & $\boldsymbol{k}_{\boldsymbol{z}}(\mathbf{k N} / \mathbf{m})$ & LD ratio \\
\hline A1 & -47.1 & & -0.1623 & 196.9 & 220.0 & 81.6 \\
B1 & -35.9 & 13.24 & -0.0744 & 162.1 & 309.2 & 178.0 \\
C1 & -33.9 & & -0.0589 & 151.2 & 343.0 & 224.8 \\
\hline
\end{tabular}
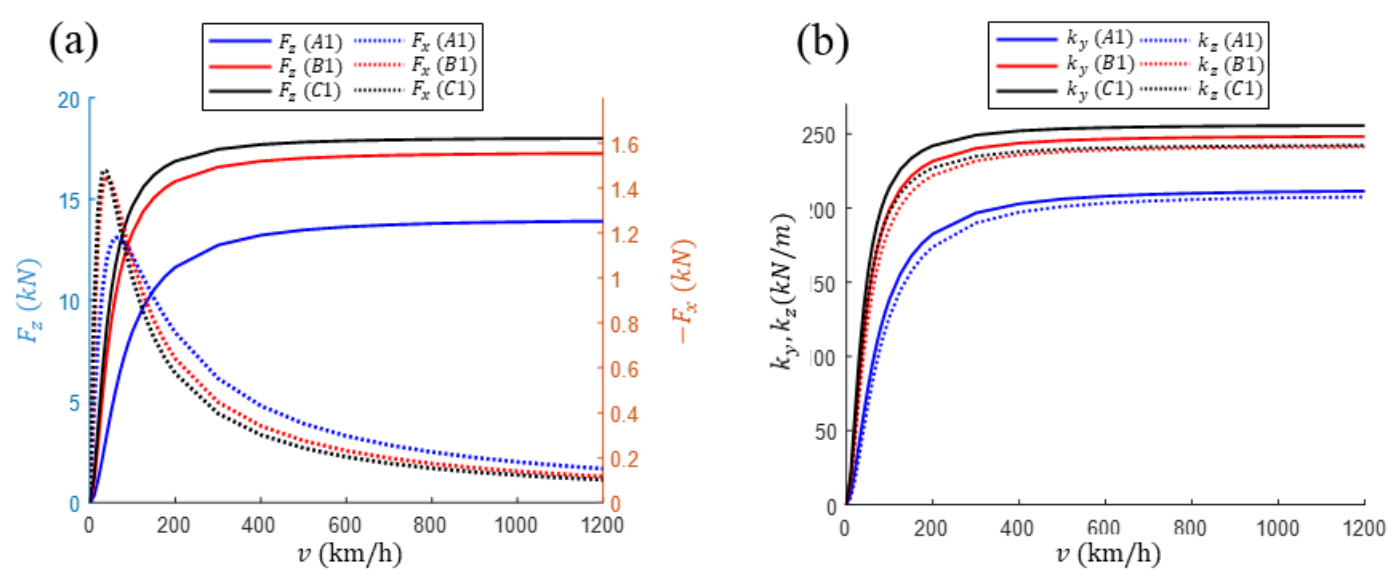

Figure 7. Comparison of characteristics of the designs with respect to changes in the driving velocity; (a) lift and drag forces; and (b) horizontal and vertical stiffnesses. 
Table 4. Comparison of magnetic levitation/guidance characteristics of three designs when magnetically levitated pods are moving at $\Delta \mathbf{z}=-50 \mathrm{~mm} \mathrm{~mm}$ for velocities $v_{h}$ and $v_{l}$.

\begin{tabular}{ccccccccc}
\hline Design & $\Delta \boldsymbol{z}(\mathbf{m m})$ & $\boldsymbol{v}$ & $\boldsymbol{F}_{\boldsymbol{z}}(\mathbf{k N})$ & $\boldsymbol{F}_{\boldsymbol{x}}(\mathbf{k N})$ & $\boldsymbol{k}_{\boldsymbol{y}}(\mathbf{k N} / \mathbf{m})$ & $\boldsymbol{k}_{\boldsymbol{z}}(\mathbf{k N} / \mathbf{m})$ & LD ratio & $\boldsymbol{F}_{\boldsymbol{z} \boldsymbol{r}}(\boldsymbol{\%})$ \\
\hline \multirow{2}{*}{ A1 } & & $v_{h}$ & 13.87 & 0.1810 & 210.8 & 206.8 & 76.6 & 75.9 \\
& & $v_{l}$ & 10.52 & 0.9104 & 167.5 & 157.3 & 11.6 & \\
B1 & \multirow{2}{*}{50} & $v_{h}$ & 17.24 & 0.1394 & 247.9 & 240.8 & 123.7 & 87.4 \\
& & $v_{l}$ & 15.07 & 0.8102 & 221.5 & 210.7 & 18.6 & \\
C1 & & $v_{h}$ & 17.99 & 0.1226 & 255.3 & 241.8 & 146.7 & 90.0 \\
\hline
\end{tabular}

\subsection{Model Validation with the FEM Results}

The EIM that can significantly improve the computational speed should be validated for its accuracy. First, the analyzed results for different designs were compared with the analysis results using FEM, to validate the applicability of the EIM to general EDS coil analysis. Thereafter, the analysis accuracies of the three selected designs were validated against the analysis results using FEM, followed by the accuracy of the EIM with respect to changes in $P_{e}$, which represents the number of adjacent coil pairs to be modeled. In addition, the accuracy of the induced EMF calculation with respect to changes in the number of loops was examined. For the validation analysis, $P_{e}=3$ for the EIM and $n_{\text {turn }}=N_{\text {turn }}$ for the EMF computation were applied. Moreover, the errors of the analyzed results using the EIM and FEM were evaluated by the following mean absolute percentage error (MAPE) for a total of $N$ data points:

$$
\operatorname{MAPE}(\%)=\frac{1}{N} \sum_{i=1}^{N}\left|\frac{f_{i}-\bar{f}_{i}}{\bar{f}_{i}}\right| \times 100
$$

where $f_{i}$ and $\bar{f}_{i}$ are the data at the $i$-th data point computed by the EIM and FEM, respectively.

To validate the EIM, various designs that changed $N_{t u r n}$ or $L_{z c}$ in Designs A1, B1, and C1 were considered. When driving at velocity $v_{h}$, the lift forces calculated at the maximum vertical displacement $P_{z m}$ for each design were compared with the FEM analysis results, as shown in Figure 8, where the errors evaluated for the FEM results were plotted on the right axis. The $F_{z}$ evaluated for a total of 61 designs revealed that the values were in good agreement with respect to the design feature changes, and that the errors in all the cases were within $1 \%$, thus confirming that the EIM could be effectively utilized for various coil designs with varying sizes and $N_{\text {turn }}$ values, among other parameters.
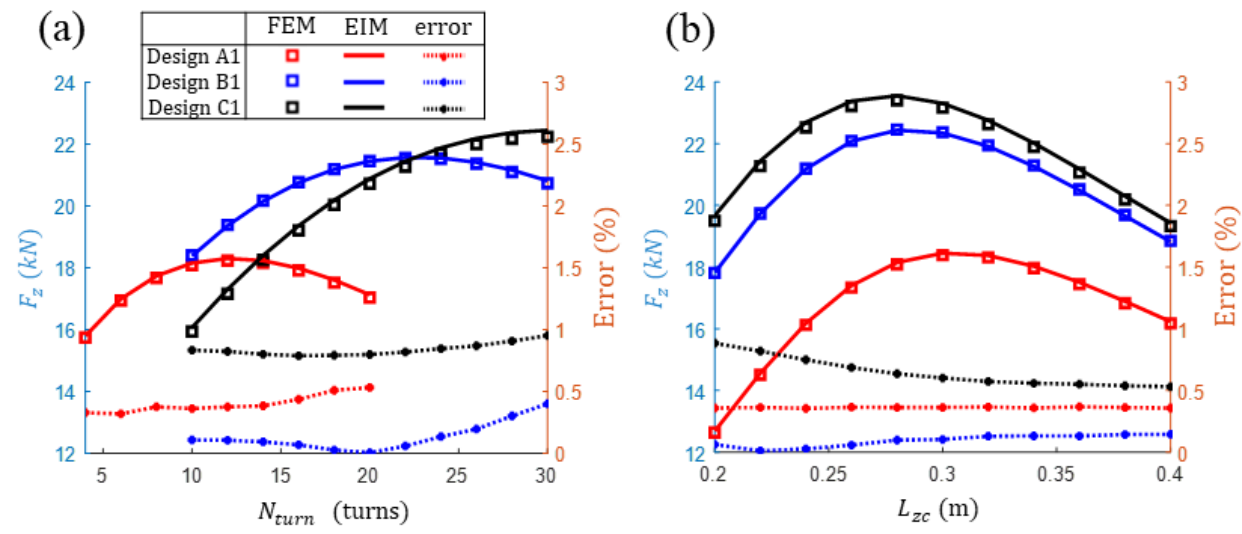

Figure 8. Comparison of the lift forces analyzed using the FEM and EIM for different designs with respect to the (a) number of turns $N_{\text {turn }}$ and (b) vertical coil height $L_{z c}$. 
(a)
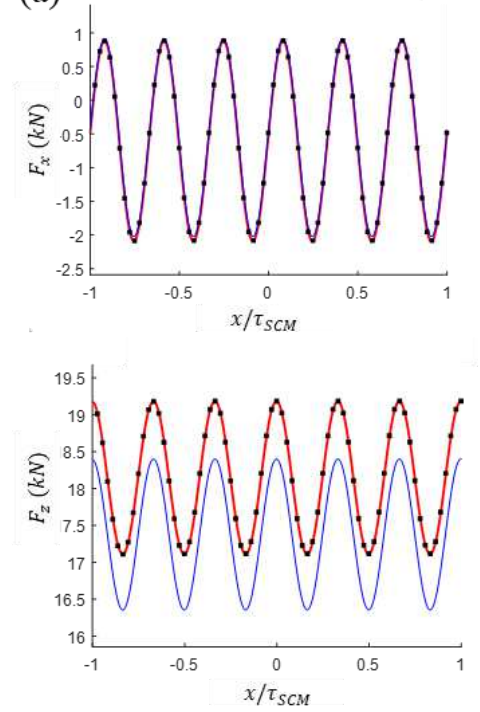

(b)
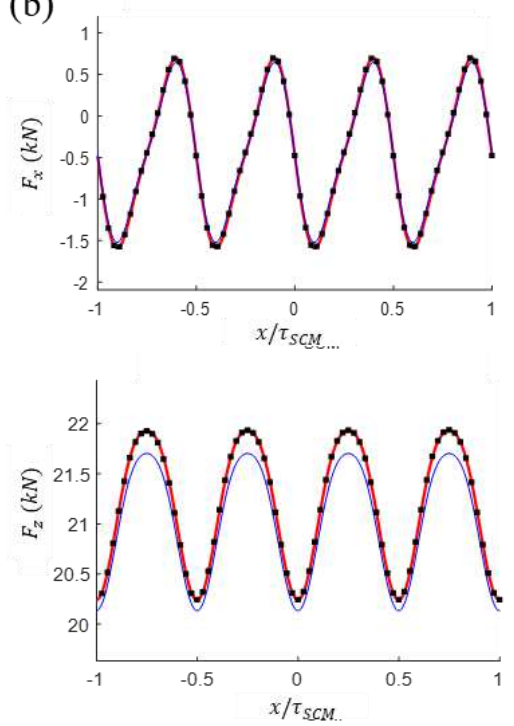
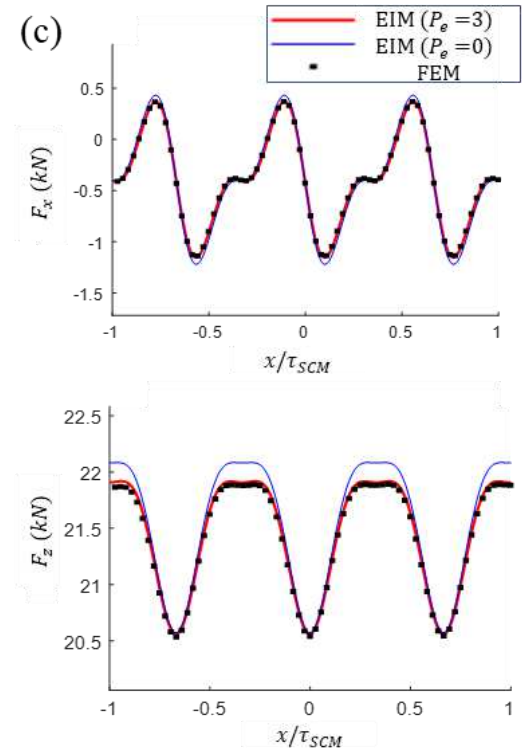

Figure 9. Comparison of drag and lift force responses acting on the SCM driving in the $P_{z m}$ for the analysis results of Design (a) A1, (b) B1, and (c) C1 based on the FEM and EIM.

(a)
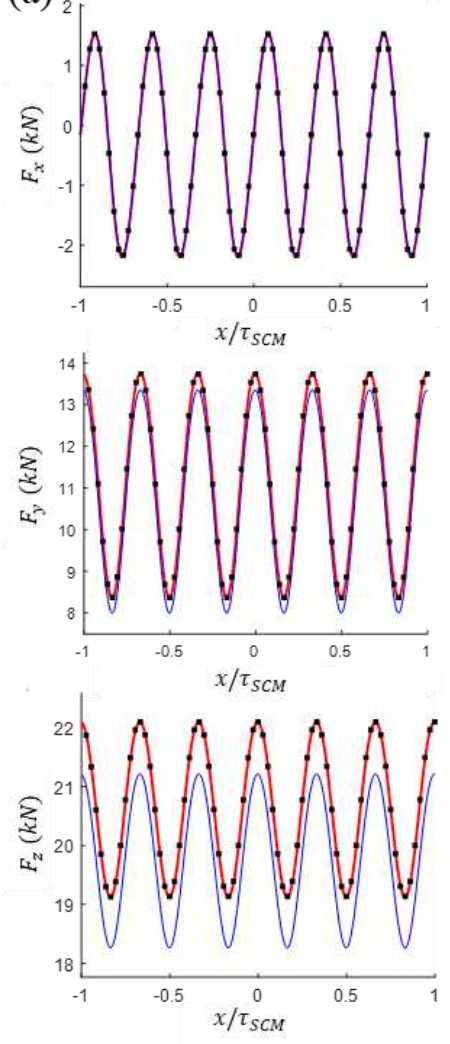

(b)
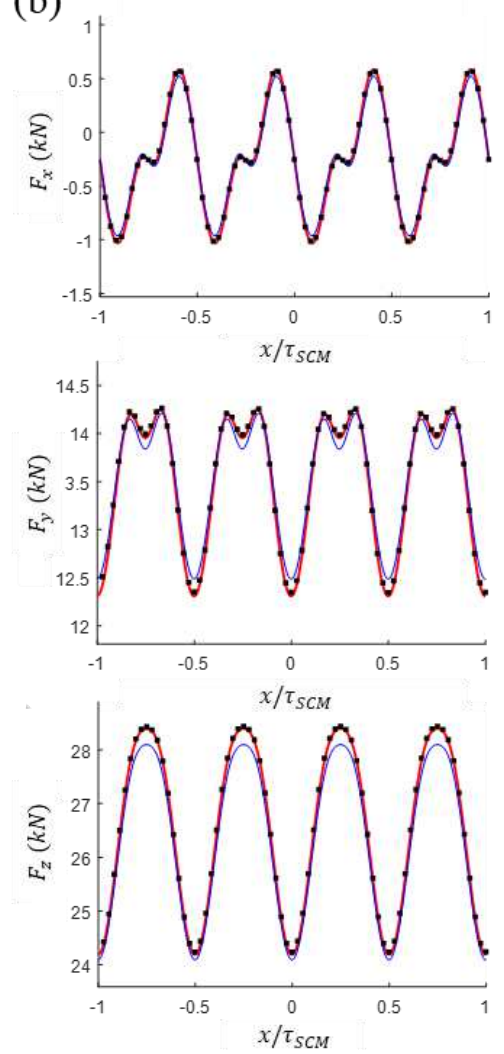

(c)
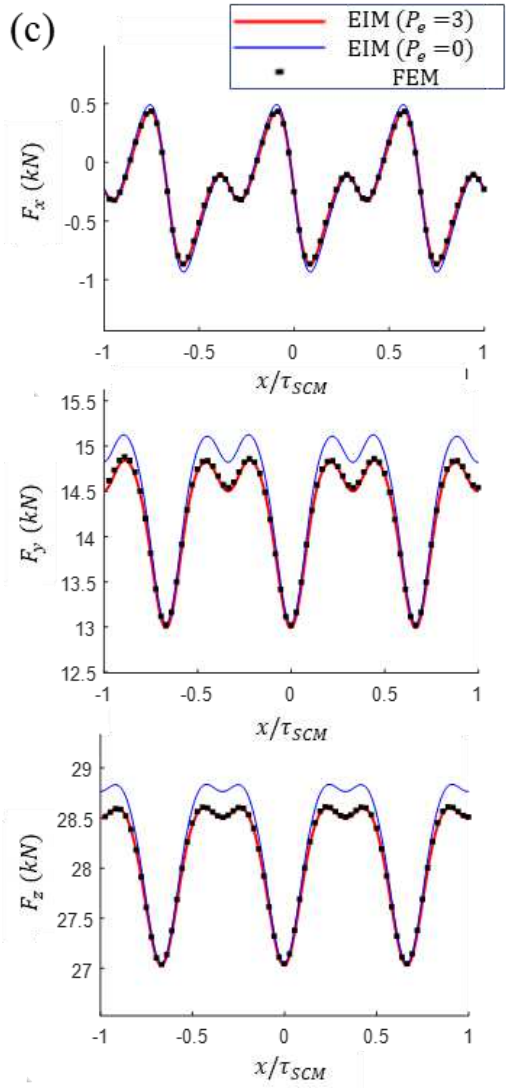

Figure 10. Comparison of drag, guidance, and lift force responses acting on the SCM driving in $P_{z m}$ for the analysis results of Design (a) A1, (b) B1, and (c) C1 based on the FEM and EIM. 
For a more detailed validation of the model, the analysis results for the drag, guidance, and lift force responses of the three designs were compared with the FEM results. Figures 9 and 10 present a comparison of the force responses at the $P_{z m}$ and the maximum horizontal displacement $P_{y m}=\left(-\Delta \mathrm{y}_{\max },-\Delta \mathrm{z}_{\max } / 2\right)$ with the FEM results, and the calculated results for $P_{e}=0$ and $P_{e}=3$ are presented to compare the accuracy of the model according to the $P_{e}$ values. The analysis results for $P_{e}=3$ were consistent with the FEM analysis results in all cases. The analysis with $P_{e}=0$ yielded a certain level of error; however, the waveform was similar. The computational errors of the EIM with respect to changes in the $P_{e}$ values are listed in Table 5. A relatively small drag resulted in a large number of near-zero data; therefore, error calculations were evaluated more than the actual difference. Although $P_{e}=0$ exhibited satisfactory analysis results and only included the inductance effects in electrically connected null-flux coils, relatively large errors were observed in Designs A1 and C1. A high accuracy was achieved at $P_{e}=1$, which contained only the inductance effects of the closest pair of null-flux coils. In general, with an increase in $P_{e}$, the analysis error decreased. As $P_{e}$ increased, the reaction forces converged to certain values, and the mutual inductance decreased abruptly as the coil distance increased. Thus, it is effective in computing to limit $P_{e}$ to a certain level.

Table 5. Comparison of analysis accuracies for three models with respect to various $P_{e}$ values in the EIM.

\begin{tabular}{|c|c|c|c|c|c|c|}
\hline \multirow[t]{2}{*}{ Design } & \multirow[t]{2}{*}{$P_{e}$} & \multicolumn{2}{|c|}{ Error $(\%)$ in $P_{z m}$} & \multicolumn{3}{|c|}{ Error $(\%)$ in $P_{y m}$} \\
\hline & & $F_{x}$ & $F_{Z}$ & $F_{x}$ & $F_{y}$ & $F_{Z}$ \\
\hline \multirow[t]{4}{*}{ A1 } & 0 & 14.665 & 4.258 & 4.937 & 3.541 & 4.259 \\
\hline & 1 & 6.252 & 0.101 & 4.974 & 1.016 & 0.101 \\
\hline & 2 & 6.191 & 0.078 & 4.990 & 1.065 & 0.130 \\
\hline & 3 & 6.213 & 0.078 & 4.991 & 1.084 & 0.152 \\
\hline \multirow[t]{4}{*}{ B1 } & 0 & 14.079 & 0.795 & 16.328 & 0.671 & 0.888 \\
\hline & 1 & 8.864 & 0.060 & 9.012 & 0.618 & 0.103 \\
\hline & 2 & 8.872 & 0.034 & 9.077 & 0.591 & 0.157 \\
\hline & 3 & 8.877 & 0.034 & 9.100 & 0.590 & 0.160 \\
\hline \multirow[t]{4}{*}{ C1 } & 0 & 38.319 & 0.592 & 20.604 & 1.238 & 0.496 \\
\hline & 1 & 8.148 & 0.158 & 6.791 & 0.807 & 0.055 \\
\hline & 2 & 7.452 & 0.140 & 7.020 & 0.794 & 0.049 \\
\hline & 3 & 7.461 & 0.140 & 7.018 & 0.794 & 0.049 \\
\hline
\end{tabular}

The computational accuracies of the induced EMF with respect to changes in the number of closed-loops $n_{\text {turn }}=n_{w} \times n_{t}$ were compared. When driving in $P_{z m}$ and $P_{y m}$ at $v_{h}$, the calculated force errors of Design B1 for the FEM results are shown in Figure 11. The number of closed loops was changed from a single loop to $n_{w}=9, n_{t}=2$; which was the same as the actual number of turns in Design B1. In addition, the simplest shape, namely, the rectangular single loop without a round corner, as denoted by rect, was considered. As mentioned previously, errors for $F_{x}$ were evaluated as larger than the actual values. The lift force error for the rect loop was up to $5 \%$ and $3.4 \%$ for the single loop. For $n_{w}=3$ or a higher number of loops, the errors in the lift/guidance forces were sufficiently low (within $0.5 \%$ ), and the forces for $n_{t}=2$ exhibited higher accuracies. Therefore, the coil shape in the EMF computation can be accurately explained, even at a lower number of turns $n_{\text {turn }}$ than the actual value $N_{\text {turn }}$. 


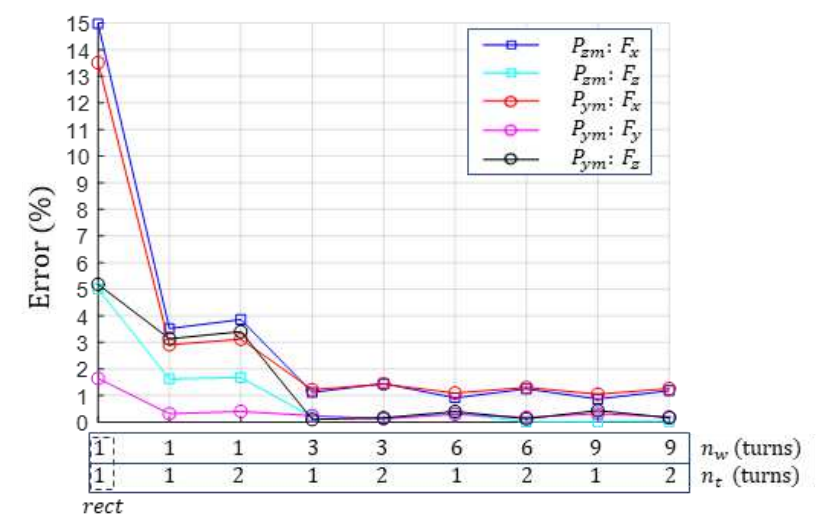

Figure 11. Comparison of errors for analyzed forces with respect to changes in the number of loops in Design B1.

\section{Experimental Validation}

A small-scale testbed constructed for the LSM/EDS combination test of the Hyperloop was utilized to experimentally validate the EIM model, as shown in Figure 12. In the test bed, a pod was accelerated by the LSM installed in the section with a length of $60 \mathrm{~m}$, and then braked by the eddy current induced on the aluminum plate in the rear section. In the experiment, two types of pods with the same pole pitch were used: the first with $150 \mathrm{kAt}$ of SCMs, and the second with N52 grade neodymium PMs. Moreover, the PM pole was customized to segment 78 block PMs, to reduce the mass of the PM pod. In the test bed, 10 sets of fabricated null-flux coils of an initial design were installed at a distance of 5.4 m near the maximum velocity section, and induced currents measured by four current sensors (LF 1005-S) attached to the sixth null-flux coil were used for validation. The tests were conducted at velocities of $v_{S C M}$ and $v_{P M}$ velocities for the SCM and PM pods, respectively. The detailed parameters related to the experiment are summarized in Table 6.

(c)

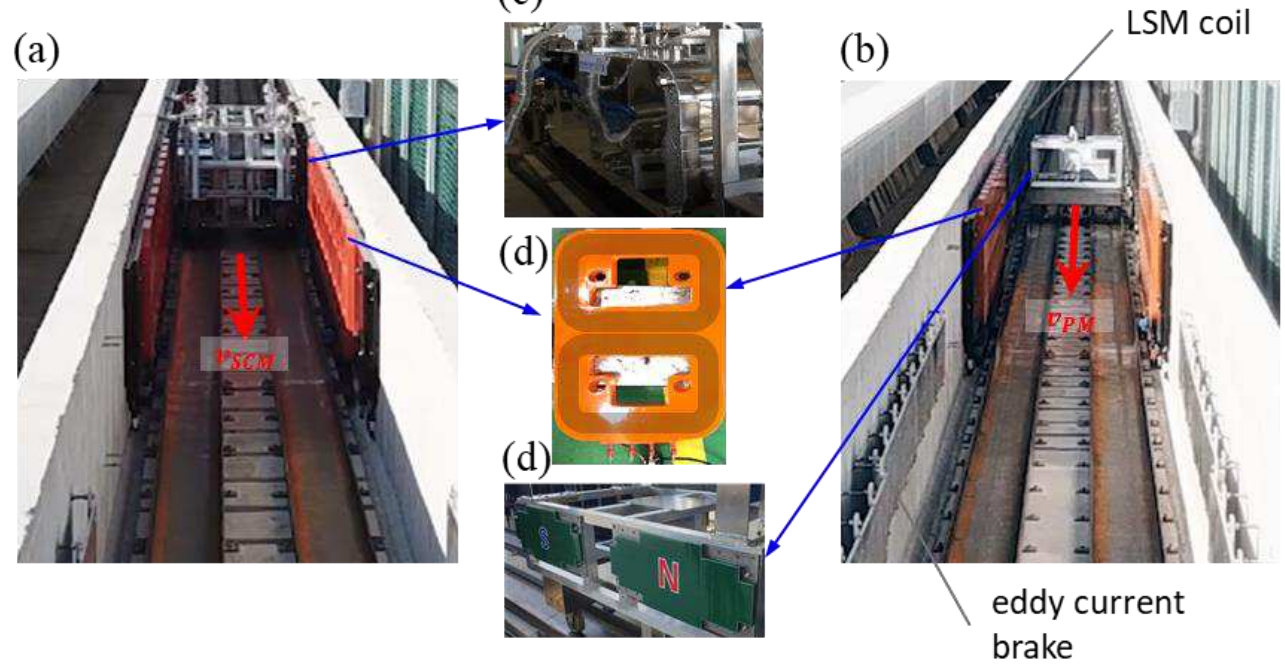

Figure 12. Induced current experiment of a null flux coil in a small test bed for EIM validation: (a) SCM pod, (b) PM pod, (c) SCM module, (d) null-flux levitation coil, and (d) customized PM poles. 
Table 6. Experimental parameters of PM bogie and null-flux coils in small-scale testbed.

\begin{tabular}{cccc}
\hline & Parameter & Value & Unit \\
\hline & The PM block size, $\mathrm{W} \times \mathrm{D} \times \mathrm{H}$ & $0.04 \times 0.04 \times 0.02$ & $\mathrm{~m}^{3}$ \\
The PM grade & N52 & - \\
$\mathrm{PM}$ & Number of PMs per pole & 78 & - \\
& Back iron thickness, $d_{t}$ & 0.005 & $\mathrm{~m}$ \\
& Pole pitch, $\tau_{P M}$ & 0.81 & $\mathrm{~m}$ \\
& Experiment velocity, $v_{P M}$ & $13.9(50)$ & $\mathrm{m} / \mathrm{s}(\mathrm{km} / \mathrm{h})$ \\
\hline \multirow{4}{*}{$\mathrm{SCM}$} & Coil size, $L_{(S C M) x} \times L_{(S C M) z}$ & $0.6 \times 0.3$ & $\mathrm{~m}^{2}$ \\
& Magnetomotive force, MMF & 150 & $\mathrm{kAt}$ \\
& Pole pitch, $\tau_{S C M}$ & 0.81 & $\mathrm{~m}$ \\
& Experiment velocity, $v_{S C M}$ & $13.1(47)$ & $\mathrm{m} / \mathrm{s}(\mathrm{km} / \mathrm{h})$ \\
\hline \multirow{4}{*}{ Common } & Number of poles, $N_{p o l e}$ & 4 & - \\
& Pitch ratio, $\tau_{c} / \tau_{S C M}$ & $2 / 3$ & - \\
& Levitation coil $L_{x c} \times L_{z c}$ & $0.426 \times 0.3$ & $\mathrm{~m}^{2}$ \\
& Number of turns, $N_{t u r n}=N_{w} \times N_{t}$ & $N_{w}=14, N_{t}=2$ & - \\
& Air gap, $g_{a i r}$ & 0.05 & $\mathrm{~m}$ \\
& Thickness of the levitation coil, $L_{t}$ & 0.04 & $\mathrm{~m}$ \\
& Wire cross sectional area, $A_{w}=c_{w} \times c_{t}$ & $6 \times 15$ & $\mathrm{~mm}^{2}$ \\
\hline
\end{tabular}

The currents induced by the SCM pod were measured at $P_{z 1}=(0,-30 \mathrm{~mm})$ and $P_{z 2}=(0,-60 \mathrm{~mm})$; and the currents induced by the PM pod were measured at $P_{z 2}$ and $P_{y}=(-30 \mathrm{~mm},-60 \mathrm{~mm})$. In addition, to reduce the measurement displacement error, all the induced current data were subtracted from the values measured at $(0,0)$. As shown in Figure 13, the measured and analyzed currents at two different displacements for each pod were compared at the relative positions between the center positions of the pod and the measuring null-flux coil, and $k=1-4$ were assigned to the four coils of the null-flux coil from the top left to the bottom right.

Based on the results of the EIM validation, it was confirmed that the induced currents measured in the experiment and those calculated using the model were generally consistent with highly similar patterns. When the measurement results of $P_{z 1}$ and $P_{z 2}$ moving in the horizontal center were first verified, the induced currents in the coils at the same vertical position were expected to be the same as those shown in the EIM results. However, the measured results revealed a difference of approximately 10-15\% for the maximum peak. In addition, given that the induced currents in the coils on the same side were similar, the actual vertical displacement on the left side of the pod was several millimeters lower than that on the right side. In addition, there was a difference of approximately $10-15 \%$ between the maximum peaks of the measured data and the maximum peaks of the analysis results. In the test of the PM pod at $P_{y}$, which exhibited a horizontal displacement, the measured currents of the four coils exhibited different patterns due to the presence of guidance currents. The patterns at $P_{y}$ were highly similar to the analysis results; whereas, the measured and analyzed data differed by approximately $18 \%$ for the maximum peak value.

There are several possible reasons for the difference in magnitude between the experimental data and analysis results. The first is the driving position errors due to the horizontal and vertical guideway irregularities. In addition, the actual induced current may have been low due to the contact resistance at the null-flux connection terminal. Different from the PM pod, where the velocity of the measured induced current waveform was in good agreement with the analysis result, the SCM pod exhibited a small difference of approximately $30 \mathrm{~mm}$ for the zero intersection, which can be attributed to the pole position errors of the SCMs. From a comparison of the maximum induced current and averaged lift force analyzed at $P_{z 2}$ with 
the same displacement of the two pods; the SCM pod with an MMF of 150 kAt yielded 296.3 A and 3.0 $\mathrm{kN}$, respectively; and the PM pod yielded $84.7 \mathrm{~A}$ and $0.24 \mathrm{kN}$, respectively. Thus, the MMF of the PM pod was estimated as $43 \mathrm{kAt}$. However, considering that the measured induced currents were slightly lower in the actual experiment, the actual lift and guidance forces were slightly lower than expected.

(a)

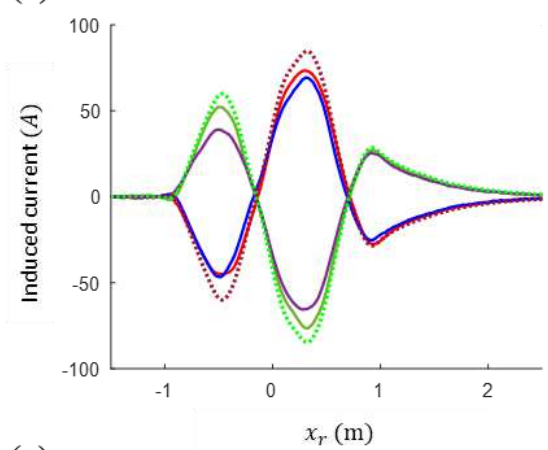

(c)

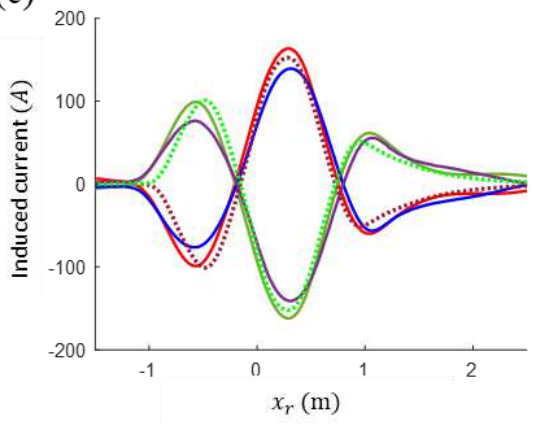

(b)

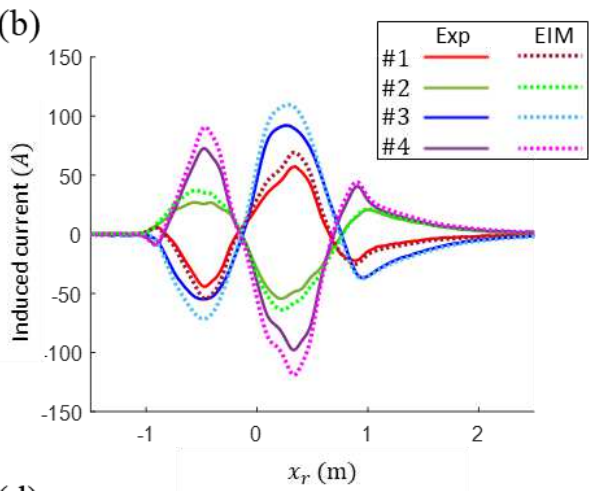

(d)

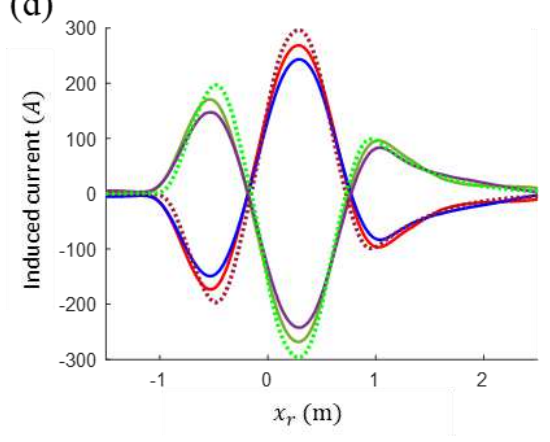

Figure 13. Comparison between the calculated induced current and the data measured by the experiment to validate EIM: induced current in (a) $P_{z 2}$ and (b) $P_{y}$ of the PM pod, and induced current in (c) $P_{z 1}$ and (d) $P_{z 2}$ of the SCM pod.

\section{Conclusions}

In this paper, an equivalent inductance model is presented for the rapid and accurate analysis of the EDS system. The EIM, which can explain the magnetic coupling effects of adjacent coils as an equivalent inductance, allows for system equations with decoupled LR equations for isolated coils. With the proposed EIM, we designed null-flux coils for SC-EDS in Hyperloop, resulting in three designs with satisfactory levitation forces and improved horizontal stiffnesses, and the characteristics of the designs were compared. In addition, the EIM exhibited a considerably high error accuracy within $1 \%$ when compared with the FEM analysis results for various designs. Using two types of moving pods, namely, SCMs and PMs on a smallscale testbed, the measured current waveforms on the levitation coils were in good agreement with the analysis results. However, several differences in the wave peak can be attributed to the position error of the pod or contact resistances of the coils. A rapid and accurate analysis of the proposed EIM can be effectively utilized for the general analysis and design of coil-based EDS levitation systems, and multiphysics simulations such as the heat transfer analysis of coils and vibration analysis during magnetic levitation.

\section{References}


1. Hyperloop Alpha. Available online:

https://www.tesla.com/sites/default/files/blog_images/hyperloop-alpha.pdf (accessed on 1 September 2021).

2. Hamad, A., Izabela, K. \& John P. HYPERLOOP-PREDICTION OF SOCIAL AND PHYSIOLOGICAL COSTS. Trans. Syst. Tech. (2020).

3. Gkoumas, K. \& Christou, M. A Triple-Helix Approach for the Assessment of Hyperloop Potential in Europe. Sustainability 12.19, 7868 (2020).

4. Gkoumas, K. Hyperloop Academic Research: A Systematic Review and a Taxonomy of Issues. Appl. Sci. 11.13, 5951 (2021).

5. Nøland, J. K. Prospects and challenges of the hyperloop transportation system: A systematic technology review. IEEE Access (2021).

6. Mitropoulos, L. et al. The Hyperloop System and Stakeholders: A Review and Future Directions. Sustainability 13.15, 8430 (2021).

7. Shanghai Maglev Transportation Development Co.,Ltd Website. Available online: http://www.smtdc.com/en (accessed on 1 September 2021).

8. Central Japan Railway Company Website. Available online: https://scmaglev.jr-central-global.com (accessed on 1 September 2021).

9. Sotelo, G. G. et al. A full scale superconducting magnetic levitation (MagLev) vehicle operational line. IEEE Trans. Appl. Supercond. 25.3, 1-5 (2014).

10. Deng, Z. et al. A high-temperature superconducting maglev-evacuated tube transport (HTS MaglevETT) test system. IEEE Trans. Appl. Supercond. 27.6, 1-8 (2017).

11. Hardt Website. Available online: https://hardt.global/technology-development/ (accessed on 1 September 2021).

12. Zeleros Website. Available online: https://zeleros.com/ (accessed on 1 September 2021).

13. Transpod Website. Available online: https://www.transpod.com/technology-demonstrator/ (accessed on 1 September 2021).

14. Nevono Website. Available online: https://www.nevomo.tech/en/ (accessed on 1 September 2021).

15. Hyperloop Transportation Technologies Website. https://www.hyperlooptt.com/technology/ (accessed on 1 September 2021).

16. Virgin Hyperloop website. https://virginhyperloop.com/

17. Choi, S. Y. et al. Sub-sonic linear synchronous motors using superconducting magnets for the hyperloop. Energies 12.24, 4611 (2019).

18. Lee, J. et al. Development of the Reduced-Scale Vehicle Model for the Dynamic Characteristic Analysis of the Hyperloop. Energies 14.13, 3883 (2021).

19. Lim, J. et al. Design optimization of a $2 \mathrm{G} \mathrm{HTS} \mathrm{magnet} \mathrm{for} \mathrm{subsonic} \mathrm{transportation.} \mathrm{IEEE} \mathrm{Trans.} \mathrm{Appl.}$ Supercond. 30.4, 1-5 (2020).

20. Mun, J. et al. Thermal and Electromagnetic Performance Evaluation of REBCO Magnet With Solid Nitrogen Thermal Battery for Maglev Train. IEEE Trans. Appl. Supercond. 31.5, 1-5 (2021).

21. Powell J. R., Jr., Danby, G. T. Electromagnetic Inductive Suspension and Stabilization System for a Ground Vehicle. U.S. Patent No. 3,470,828. 7 Oct. 1969

22. Hieronymus, H., Miericke, J., Pawlitschek, F. \& Rudel, M. Experimental study of magnetic forces on normal and null flux coil arrangements in the inductive levitation system. Appl. Phys. 3, 359-366 (1974).

23. Miericke, J. \& Urankar, L. Theory of electrodynamic levitation with a continuous sheet track-Part I. Appl. Phys. 2, 201-211 (1973).

24. Lever, J. H. Technical assessment of maglev system concepts. Final report. No. AD-A-358293/XAB.; CRREL-SR-98-12. Cold Regions Research and Engineering Lab,; Hanover, NH (United States), 1998. 
Available online: https://rosap.ntl.bts.gov/view/dot/42287/dot_42287_DS1.pdf? (accessed on 3 August 2020).

25. Lim, S., Kim, D., Yoo, J. \& Park, N. C. A method of induced current estimation of EDS type maglev using 3D transient EM analysis. Trans. Korean Soc. Mech. Eng. 43, 253-259 (2019).

26. Guo, Z., Li, J. \& Zhou, D. Study of a null-flux coil electrodynamic suspension structure for evacuated tube transportation. Symmetry 11, 1239 (2019).

27. Gong, T. et al. 3-D FEM Modeling of the Superconducting EDS Train With Cross-Connected FigureEight-Shaped Suspension Coils. IEEE Transactions on Applied Superconductivity 31.3 (2021): 1-13.

28. Burkhardt, E. E., Schwartz, J. \& Nakamae, S. Analysis of superconducting magnet (SCM)-ground coil interactions for EDS Maglev coil configurations. IEEE Trans. Appl. Supercond. 3.1, 430-433 (1993).

29. He, J. L., Rote, D. M., Coffey, H. T. Applications of the dynamic circuit theory to maglev suspension systems. IEEE Trans. Magn. 29, 4153-4164 (1993).

30. Davey, K. R. Designing with null flux coils. IEEE Trans. Magn. 33.5, 4327-4334 (1997).

31. He, J. L., Coffey, H. T. \& Rote, D. M. Analysis of the combined maglev levitation, propulsion, and guidance system. IEEE Trans. Magn. 31.2, 981-987 (1995).

32. Cai, Y. et al. Semianalytical calculation of superconducting electrodynamic suspension train using figure-eight-shaped ground coil. IEEE Trans. Appl. Supercond. 30.5, 1-9 (2020).

33. Semail, E., Bouscayrol, A. \& Hautier., J. -P. Vectorial formalism for analysis and design of polyphase synchronous machines. EPJ Appl. Phys. 22.3, 207-220 (2003).

34. Sellam, A. et al. A Vectorial modeling for the Permanent Magnet Synchronous Machine (polyphase) based on multimachine approach. Int. J. Electr. Eng. Inform. 5.1, 67 (2013).

35. Lim, J. et al. Design Model of Null-Flux Coil Electrodynamic Suspension for the Hyperloop. Energies 13.19, 5075 (2020).

36. Yoon, R. et al. Capsule Vehicle Dynamics Based on Levitation Coil Design Using Equivalent Model of a Sidewall Electrodynamic Suspension System. Energies 14.16, 4979 (2021).

\section{Acknowledgements}

This work was supported by "Core Technology Development of Subsonic Capsule Train" of the Korea Railroad Research Institute under Grant PK2101A1, Republic of Korea.

\section{Author contributions}

Conceptualization and methodology, J.L. and S.C.; formal analysis and validation, J.L., J.-H. L and Y.J.O.; experiment and validation, J.-M.J., Y.J.O., S.C., J.L. and C.-Y.L.; supervision and project administration, C.-Y.L. and K.-S.L. All authors have read and agreed to the published version of the manuscript.

\section{Competing interests}

The authors declare no competing interests. 